\title{
Clinical Correlation between Perverted Nystagmus and Brain MRI Abnormal Findings
}

\author{
Won-Gue Han, Hee-Chul Yoon, Tae-Min Kim, Yoon Chan Rah, and June Choi \\ Department of Otorhinolaryngology-Head and Neck Surgery, Korea University College of Medicine, Seoul, Korea
}

Received February 22, 2016

Revised May 3, 2016

Accepted May 9, 2016

\author{
Address for correspondence \\ June Choi, $\mathrm{MD}, \mathrm{PhD}$ \\ Department of Otorhinolaryngology- \\ Head and Neck Surgery, \\ College of Medicine, \\ Korea University Ansan Hospital, \\ 123 Jeokgeum-ro, Danwon-gu, \\ Ansan 15355, Korea \\ Tel $+82-31-412-5170$ \\ Fax +82-31-412-5174 \\ E-mail mednlaw@korea.ac.kr
}

Background and Objectives: To analyze the clinical correlation between perverted nystagmus and brain magnetic resonance imaging (MRI) abnormal findings and to evaluate whether perverted nystagmus is clinically significant results of brain abnormal lesions or not. Subjects and Methods: We performed medical charts review from January 2008 to July 2014, retrospectively. Patients who were suspected central originated vertigo at Frenzel goggles test were included among patients who visited our hospital. To investigate the correlation with nystagmus suspected central originated vertigo and brain MRI abnormal findings, we confirmed whether performing brain MRI or not. Then we exclude that patients not performed brain MRI. Results: The number of patients with perverted nystagmus was 15 , upbeating was 1 and down-beating was 14. Among these patients, 5 patients have brain MRI abnormal findings. However, 2 patients with MRI abnormal findings were not associated correctly with perverted nystagmus and only 3 patients with perverted nystagmus were considered central originated vertigo and further evaluation and treatment was performed by the department of neurology. Conclusions: Perverted nystagmus was considered to the abnormalities at brain lesions, especially cerebellum, but neurologic symptoms and further evaluation were needed for exact diagnosis of central originated vertigo.

J Audiol Otol 2016;20(2):85-89

KEY WORDS: Pathologic nystagmus · Magnetic resonance imaging · Dizziness.

\section{Introduction}

Central vestibular abnormalities are most often the result of lesions of numerous pathways to the vestibulo-cerebellum or of core areas caused by infarction, hemorrhage, multiple sclerosis plaques, or rare tumors. The most important thing concerning vertigo is its differential diagnosis between peripheral vertigo and cerebrovascular or cerebellovascular abnormalities that are similar to peripheral vertigo [1-3].

As the current, one important thing to evaluate dizziness related to central or peripheral originated lesions is the determination of nystagmus with the aid of Frenzel goggles test, with distinction between voluntary nystagmus and provoked nystagmus. These examinations can be carried out by the gen-

This is an Open Access article distributed under the terms of the Creative Commons Attribution Non-Commercial License (http://creativecommons.org/licenses/by-nc/3.0/) which permits unrestricted non-commercial use, distribution, and reproduction in any medium, provided the original work is properly cited. eral doctor, not specialist [4]. Furthermore, Frenzel goggles test enable a definitive differentiation of an otologic illness like benign paroxysmal positional vertigo, vestibular neuritis, and Meniere's disease from a neurological disorder [2,5].

Although the pathophysiology of perverted head shaking nystagmus remains unclear, cerebellar abnormal lesions may induce vertical nystagmus during head shaking. Positional down beating nystagmus primarily originates from abnormal lesions of flocculonodular lobes or posterior vermis of the cerebellum $[6,7]$. Perverted head shaking nystagmus refers to vertical direction of eye movement induced by horizontal head oscillation or sustained horizontal chair rotation $[3,8]$. Therefore, perverted nystagmus spontaneously or perverted head shaking nystagmus may be useful in investigating subclinical cerebellar dysfunction [9]. Furthermore, these types of nystagmus can be easily observed at the bedside by applying Frenzel goggles [10,11].

Despite the numerous clinical studies performed on upbeat 
and downbeat nystagmus, so far the pathophysiology of the disorders has not been clarified. We studied our investigation to evaluate the association between the presence of perverted nystagmus and brain imaging work up.

\section{Subjects and Methods}

We retrospectively reviewed medical records from January 2008 to July 2014 to identify patients who complained feeling dizziness, and we selected patients suspected central vertigo at video Frenzel goggles test (perverted nystagmus, oscillopsia, etc.) in the department of otorhinolaryngologyhead and neck surgery at Korea University Ansan Hospital. To exactly evaluate causes of dizziness and rule out central originated vertigo, we strongly recommended the patients to take the brain magnetic resonance imaging (MRI). Almost all patients took the brain MRI when their symptoms were occurred within 2 weeks (mean interval: $7.8 \pm 4.3$ days), except 3 patients. To investigate the correlation with eye movement suspected central originated vertigo at Frenzel goggles test and brain MRI abnormal findings, we confined whether performing brain MRI or not. We selected 31 patients and evaluated nystagmus and brain MRI findings. In addition, we reviewed 5 patients who were diagnosed brain infarction, hemorrhage, and multiple sclerosis at department of neurology before visiting our department.

\section{Patients}

A total of 34 patients participated in this study. Among these patients, we selected 31 patients because 3 cases had no brain MRI records. The patients included 18 women and 13 men who ranged in age from 19 to 82 years (mean \pm standard deviation $=53.1 \pm 15.5$ ). At the first and follow-up visit for evaluating dizziness, all of the patients underwent Frenzel goggles test for identifying head-shaking and positional nystagmus. Furthermore, the presence of dysarthria, general weakness, gait ataxia was examined by patients' interview and several physical examination.

\section{Frenzel goggles test}

Patients were asked to sit upright with their eyes opened comfortably, spontaneous nystagmus and gaze nystagmus was measured. Positioning nystagmus was induced by the Dix-Hallpike and straight head-hanging maneuvers. At each position, the patients were instructed not to close their eyes. During measuring head shaking nystagmus, patient's eyes closed and their head tilted forward about 30 degrees to bring the horizontal semicircular canals into the plane of stimulation. The head was shaken horizontally by an examiner in a sinusoidal manner at a rate of $2.5 \mathrm{~Hz}$ with an amplitude of 10 degrees for $10 \mathrm{~s}$. After the head-shaking, the patients were instructed to open their eyes and look straight ahead. Eye movements were recorded with an infrared camera (resolution of $640 \times 480$ pixels, frame rate of $60 \mathrm{~Hz}$ ) and displayed on a computer monitor (SLMED, Seoul, Korea). The nystagmus was analyzed by otologist at the department of otorhinolaryngology-head and neck surgery at Korea University Ansan Hospital.

\section{Brain MRI}

Thirty-one out of the 34 cases had brain MRI records. Three cases refused brain MRI because of low social economic status or no time to perform brain MRI due to their own schedule. In this study, brain MRI include brain MRIdiffusion, brain MRI, brain MRI-magnetic resonance angiography (MRA), and brain MRI-internal auditory canal (MRIIAC). Brain MRI-diffusion is a commonly performed MRI sequence for evaluation of acute ischemic stroke, and is sensitive in detection of small and early infarcts. Brain MRI is also sensitive in detection of infarcts and can more exact assessment about pathologic lesions. MRA generates pictures of the arteries or aneurysms for evaluating abnormal narrowing or vessel wall dilatations. In this study, brain MRI-MRA means that brain MRI was performed with brain MRA, and brain MRI-IAC means the brain MRI focused on posterior fossa area. Seventeen cases performed brain MRI-diffusion, 4 cases performed brain MRI, 15 performed brain MRIMRA, and 2 performed brain MRI-IAC. In these cases, 4 cases performed brain MRI-diffusion with brain MRI-MRA, 1 case performed brain MRI-diffusion with brain MRI-IAC, and 1 case performed brain MRI-diffusion, brain MRI-MRA, and brain MRI-IAC.

\section{Statistical analysis}

We analyzed comparisons of the occurrences of perverted nystagmus and of brain MRI abnormal findings and odds ratio of several factors about occurring perverted nystagmus. The statistical analyses were conducted using the SPSS software (version 22.0; SPSS Inc., Chicago, IL, USA), $p<0.05$ was considered statistically significant. This study was reviewed and approved by the Korea University Ansan Hospital Institutional Review Board (AS15068).

\section{Results}

There were 31 patients (male is 13, female is 18) and mean age of patients was $53.1 \pm 15.5$ years old. We exclude 3 patients, total number of cases is 31 . The number of patients with perverted nystagmus is 15 , up-beating is 1 and down-beating 


\section{is 14 (Table 1).}

Among these patients, 5 patients have Brain MRI abnormal findings. However, 2 MRI abnormal findings (patient No. 14,15 ) were not associated correctly with perverted nystagmus. 3 patients with perverted nystagmus (patient No. 11, $12,13)$ were considered central originated vertigo and further evaluation and treatment was performed by department of neurology (Fig. 1). In No. 11 patient, although the reading of brain MRI was old infarction, we treated anti-coagulant therapy in accordance with the acute left cerebellar infarction after the consultation of department of neurology. The reason was that her symptoms onset was 1 week ago and we didn't rule out the progression of old infarction. Despite the brain MRI-diffusion reading of No. 13 patient was old lacunar infarction at left cerebellum, we consulted to department of neurology and didn't rule out acute infarction because perverted nystagmus was persisted, dizziness was not improved, and acute lacunar infarction could be seen low signal intensity at brain MRI-diffusion. Unfortunately, No. 13 patient refused further work up and re-admitted at our department of neurology due to left mid brain and both cerebellar infarction with vertebral artery stenosis after 5 months.

Table 1. Data of 15 patients who had perverted nystagmus at Frenzel goggles test.

\begin{tabular}{cccll}
\hline No. & Sex & Age & Frenzel goggles test & \\
\hline 1 & M & 19 & SN, HST: up-beating & I: No abnormalities \\
2 & M & 39 & SN, HST: down-beating & I: No abnormalities \\
3 & M & 56 & HST: down-beating & I: No abnormalities \\
4 & F & 58 & HST: down-beating & D, I: No abnormalities \\
5 & M & 41 & HST: down-beating & D, I, IAC: No abnormalities \\
6 & F & 44 & HST: down-beating & D, I: No abnormalities \\
7 & F & 65 & SN, HST: down-beating & D: No abnormalities \\
8 & F & 38 & HST: down-beating & D, IAC: No abnormalities \\
9 & F & 80 & HST: down-beating & D: No abnormalities \\
10 & F & 67 & HST: down-beating & D: No abnormalities \\
11 & F & 75 & HST: down-beating & I: possible left cerebellar hemisphere old infarction \\
12 & F & 61 & HST: down-beating & I: acute ischemic infarction at left cerebellar hemisphere \\
& & & & segmental severe stenosis at left distal vertebral artery \\
13 & $M$ & 58 & HST: down-beating & acute ischemic infarction at left pons \\
14 & F & 81 & HST: down-beating & I: suspicious mild focal stenosis at left MCA \\
15 & F & 42 & HST: down-beating & D: possible calcification at right globus pallidus
\end{tabular}

D: brain MRI-diffusion, I: Brain MRI or Brain MRI-MRA. SN: spontaneous nystagmus, HST: head shaking test, MRI: magnetic resonance imaging, MRA: magnetic resonance angiography, IAC: Internal auditory canal, MCA: middle cerebral artery

Fig. 1. Analysis of patients. We analyzed total 34 patients initially. Among 34 patients, 3 patients were excluded because they had no brain MRI records. Among 31 selected patients, 15 patients were shown perverted nystagmus at Freznel goggles test, 1 patient had up-beating nystagmus and 14 patients had down-beating nystagmus. Among 14 patients with down-beating nystagmus, 5 patients were shown abnormal findings at brain MRI records and 3 patients' abnormal findings at brain MRI were considered central lesion associated dizziness and nystagmus.

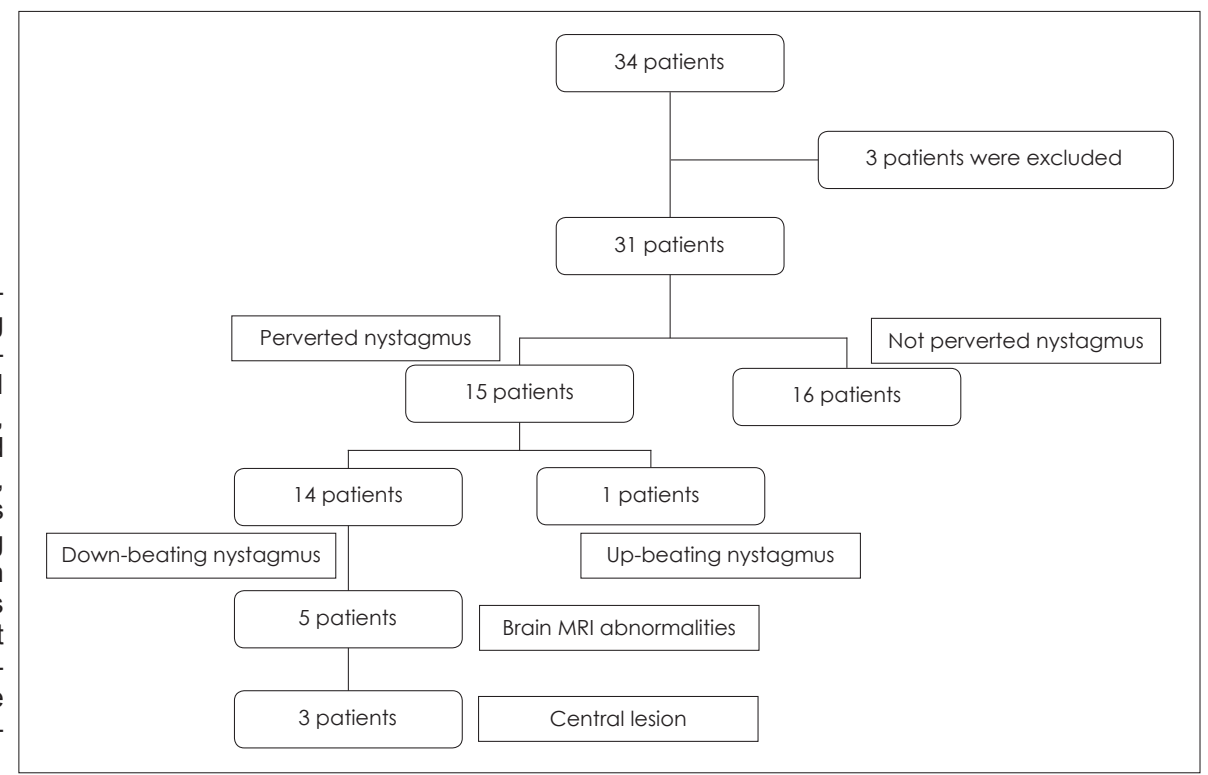


Table 2. Data of 5 patients who were diagnosed brain abnormalities at department of neurology

\begin{tabular}{ccll}
\hline Sex & Age & \multicolumn{1}{c}{ Frenzel goggles test } & \multicolumn{1}{c}{ Brain MRI } \\
\hline$M$ & 82 & No abnormalities & I: right cerebellar peduncle infarct \\
$F$ & 56 & No abnormalities & I: multiple sclerosis \\
$M$ & 53 & No abnormalities & I: both cerebellar, pontine and medullar atrophy \\
$M$ & 59 & HST: nystagmus to left & I: moderate focal stenosis, both proximal AICA and right distal vertebral artery \\
$M$ & 58 & Left D-H: nystagmus to left & D: a tiny nodular acute infarction in right cerebellum \\
& & & I: a focal mild right proximal PICA stenosis \\
\hline
\end{tabular}

D: Brain MRI-diffusion, I: Brain MRI or Brain MRI-MRA. HST: head shaking test, D-H: Dix-Hall pike test, AICA: anterior inferior cerebellar artery, PICA: posterior inferior cerebellar artery, MRA: magnetic resonance angiography

Among 15 patients who had spontaneous perverted nystagmus or perverted nystagmus at headshaking, we considered 3 patients with central lesions. And we investigated further evaluation for the remaining 12 people to find out the causes of nystagmus using pure tone audiometry, speech audiometry, caloric test, saccade test, optokinetic nystagmus test, target with tracking inspection, rotatory chair test, vestibular (VEMP) evoked myogenic potential, electrocochleography, and auditory brainstem response. Six cases did not undergo the examination, 3 cases thought to be dysfunction of the vestibular nerve like vestibular neuritis, 2 cases were suspected BPPV, and one case was observed continuing up-beating nystagmus, exact diagnosis was not made.

Furthermore, 5 patients who were diagnosed brain infarction, hemorrhage (especially cerebellar lesion), and multiple sclerosis at department of neurology before visiting our department were shown no abnormal nystagmus or abnormal nystagmus and eye movement at video Frenzel goggles test, not shown perverted nystagmus (Table 2).

We also divided 31 patients into two groups. One group was composed of 15 patients with perverted nystagmus and the other group was composed of 16 patients without visualized perverted nystagmus. We verified the correlation between perverted nystagmus and hypertension (HTN), diabetes mellitus (DM), cerebrovascular accident (CVA) history in groups using statistical methods. Odds ratio at perverted nystagmus with HTN estimated 2.889, this value was not statistically significant ( $p$ value $=0.252$ ). In addition, odds ratio at perverted nystagmus with DM estimated 3.500 ( $p$ value $=$ 0.220 ), and odds ratio at perverted nystagmus with CVA history estimated $0.310(p$ value $=0.600)$, there were no statistically significant (Table 3 ).

\section{Discussion}

In the present study, one-third (5/15) of patients showing perverted nystagmus have abnormal findings at brain imaging. In these patients, except 2 patients (possible calcification at right globus pallidus and suspicious mild focal stenosis at
Table 3. Odds ratios between perverted nystagmus and HTN, DM, and CVA history

\begin{tabular}{lcc}
\hline & Odds ratio & p value \\
\hline Perverted nystagmus \& HTN & 2.889 & 0.252 \\
Perverted nystagmus \& DM & 3.500 & 0.220 \\
Perverted nystagmus \& CVA history & 0.310 & 0.600
\end{tabular}

HTN: hypertension, DM: diabetes mellitus, CVA: cerebral vascular accident

left middle cerebral artery), brain MRI abnormal findings were correlated with perverted nystagmus.

It was reported that 9 patients $(50 \%)$ at among 18 patients with anterior inferior cerebellar artery infarction showed patterns of head-shaking nystagmus that included perverted nystagmus $(n=7)$, head-shaking nystagmus in the opposite direction of spontaneous nystagmus $(n=4)$, and head-shaking nystagmus beating towards unilateral canal paresis or abnormal head impulse testing $(n=3)$ [12].

Furthermore, in another study, it was investigated spontaneous nystagmus and head-shaking nystagmus in 72 patients with isolated cerebellar infarction. Twenty eight patients had spontaneous nystagmus, 37 patients had head-shaking nystagmus including perverted nystagmus and horizontal nystagmus. Authors suggested that, perverted head-shaking nystagmus occurred in 23 patients and 22 was downbeat nystagmus [13].

In addition, it was examined 32 cases who were subjected with a perverted nystagmus. Twenty four subjects had a central disease and 8 subjects had a vestibular neuritis. Authors advocated that although the perverted nystagmus was a simple examination sign, it was important because this sign was thought to be the brain stem abnormalities [14].

In some studies, only $11 \%$ of isolated cerebellar infracted patients were shown isolated vertigo, nystagmus, and postural unsteadiness that were similar to acute peripheral vestibular disorders in other study [15-17]. Eight patients with isolated cerebellar focal infarction all presented with vertigo sensation and severe imbalance. They accentuated severe imbalance and a negative head impulse test were important factors for distinguishing focal infarction from peripheral 
vestibular dysfunction [18]. As above, for accurate diagnose cerebellar focal infarct, further study correlation like bithermal caloric tests, cervical and ocular VEMPs, and brain MRI was needed [15].

In the current study, 10 patients with perverted nystagmus had no abnormal findings in brain MRI. Among these patients, 3 patients took the brain MRI-diffusion only. Since the accuracy of brain MRI-diffusion is lower than that of brain MRIMRA or other MRI settings, we could have gained more accurate result if we carried out additional brain imaging work up.

We also examined 5 patients who were diagnosed brain infarction, hemorrhage, and multiple sclerosis etc., they had no perverted nystagmus at Frenzel goggle test. Three patients had no abnormal eye movements, 2 patients had abnormal eye movements and neurologic symptoms were observed. At this evaluation, we did not consider the duration between time of diagnosis of infarction and onset of symptoms, this factor affected results of nystagmus finding.

We examined the correlation between HTN, DM, CVA history and perverted nystagmus, however, there was no statistically significant. In general, perverted nystagmus was induced by cerebellar infarction, and cerebellum is supplied by basilar artery [7]. In fact, we should have been examined other risk factors which can cause perverted nystagmus such as angina pectoris, arrhythmia, or history of acute myocardial infarction, etc. However, we couldn't acquire enough information about exact medical history through retrospective chart review in this study.

In another study, it was studied 25 patients ( 9 men and 16 women) with clinical findings compatible with two or more infarcts in the vertebro-basilar territory. The risk factors of vertebra-basilar infarction were HTN (64\%), CVA (28\%), smoking history (28\%), DM (24\%) and atrial fibrillation (20\%) [19].

Although perverted nystagmus is known as an indicator of the central lesions, results of our study were not ideal results compared with previous other studies. First, we studied too small group for evaluating the correlation between perverted nystagmus and central lesions. Second, we did not considered time of onset of symptoms and time of diagnosis at brain ischemic stroke strictly. In this reason, we missed the period of appearing perverted nystagmus at patients with cerebellar vascular abnormalities. In order to further study, we needed greater number of patients and more exact classification criteria.

In our study, three of 15 patients showing perverted nystagmus have abnormal findings at brain imaging correlated dizziness and perverted nystagmus. As above, only perverted nystagmus does not mean exactly being of abnormal lesions of brain. In conclusion, perverted nystagmus was considered to the abnormalities at brain lesions, especially cerebellum, but neurologic symptoms and further evaluation were needed for exact diagnosis of central originated vertigo.

\section{Conflicts of interest}

The authors have no financial conflicts of interest.

\section{REFERENCES}

1) Batuecas-Caletrío Á, Yáñez-González R, Sánchez-Blanco C, González-Sánchez E, Benito J, Gómez JC, et al. [Peripheral vertigo versus central vertigo. Application of the HINTS protocol]. Rev Neurol 2014;59:349-53.

2) Kim CH, Shin JE, Song CI, Yoo MH, Park HJ. Vertical components of head-shaking nystagmus in vestibular neuritis, Meniere's disease and migrainous vertigo. Clin Otolaryngol 2014;39:261-5.

3) Minagar A, Sheremata WA, Tusa RJ. Perverted head-shaking nystagmus: a possible mechanism. Neurology 2001;57:887-9.

4) Dodt C, Zelihic E. [Vertigo and dizziness. Diagnostic algorithm from the perspective of emergency medicine]. Med Klin Intensivmed Notfmed 2013;108:41-6.

5) Michel O. [Central or vestibular vertigo? Diagnostic look through Frenzel glasses]. MMW Fortschr Med 2000;142:38-40.

6) Shimizu N. [Neurology of eye movements]. Rinsho Shinkeigaku 2000;40:1220-3.

7) Kim JS, Kim JS, Youn J, Seo DW, Jeong Y, Kang JH, et al. Ocular motor characteristics of different subtypes of spinocerebellar ataxia: distinguishing features. Mov Disord 2013;28:1271-7.

8) Strupp M, Walther LE, Eckhardt-Henn A, Franko Zeitz P. [Diagnosis of vertigo: keep an eye on central eye movement disorders]. Ophthalmologe 2013;110:31-8.

9) Baloh RW. Differentiating between peripheral and central causes of vertigo. Otolaryngol Head Neck Surg 1998;119:55-9.

10) Bertholon P, Tringali S, Faye MB, Antoine JC, Martin C. Prospective study of positional nystagmus in 100 consecutive patients. Ann Otol Rhinol Laryngol 2006;115:587-94.

11) Baba S, Fukumoto A, Aoyagi M, Koizumi Y, Ikezono T, Yagi T. A comparative study on the observation of spontaneous nystagmus with Frenzel glasses and an infrared CCD camera. J Nippon Med Sch 2004;71:25-9.

12) Huh YE, Koo JW, Lee H, Kim JS. Head-shaking aids in the diagnosis of acute audiovestibular loss due to anterior inferior cerebellar artery infarction. Audiol Neurootol 2013;18:114-24.

13) Huh YE, Kim JS. Patterns of spontaneous and head-shaking nystagmus in cerebellar infarction: imaging correlations. Brain 2011;134 (Pt 12):3662-71.

14) Toupet M, Pialoux P. [The perverted nystagmus (author's transl)]. Ann Otolaryngol Chir Cervicofac 1981;98:319-38.

15) Kim HJ, Lee SH, Park JH, Choi JY, Kim JS. Isolated vestibular nuclear infarction: report of two cases and review of the literature. $J$ Neurol 2014;261:121-9.

16) Kim HA, Lee H. Recent advances in central acute vestibular syndrome of a vascular cause. J Neurol Sci 2012;321:17-22.

17) Lee H. Isolated vascular vertigo. J Stroke 2014;16:124-30.

18) Moon IS, Kim JS, Choi KD, Kim MJ, Oh SY, Lee H, et al. Isolated nodular infarction. Stroke 2009;40:487-91.

19) Usón-Martín M, Gracia-Naya M. [Top of the basilar artery syndrome: clinico-radiological aspects of 25 patients]. Rev Neurol 1999; 28:698-701. 